\title{
EL DR. PUIGVERT Y ARCHIVOS ESPAÑOLES DE UROLOGÍA.
}

\author{
Mariano Pérez Albacete.
}

Servicio de Urología. Hospital Universitario Virgen de la Arrixaca. Murcia. España.

\begin{abstract}
Resumen.- OBJETIVO : Con motivo de celebrarse el centenario del nacimiento del Dr. D. Antonio Puigvert Gorro, deseamos conocer la contribución y la relación que tuvo con Archivos Españoles de Urología.
\end{abstract}

MÉTODOS: Se revisan todos los artículos publicados por el Dr. Puigvert en la revista, además de los que, en ella, hacen referencia a su persona y a su labor, se repasa también su obra escrita y su biografía.

RESULTADOS: Fue uno de los fundadores de la publicación, con la que mantuvo una gran colaboración hasta los años ochenta, y en ella dio a conocer importantes trabajos, que se analizan; también se resalta su aportación a la Urología además de su dedicación a la historia de la especialidad.
Mariano Pérez Albacete Servicio de Urología Hospital Universitario Virgen de la Arrixaca 30120 El Palmar. Murcia. (España) mariano.perez2@carm.es

Trabajo recibido: 25 de enero 2006
CONCLUSIONES: Constituye el Dr. Puigvert una de las figuras insignes de la Urología española, con una inmensa capacidad de trabajo y de labor escritora, en los artículos aparecidos en Archivos expone su experiencia, el resultado de sus observaciones y de sus estudios así como parte de sus innovaciones, ideas y descubrimientos, que posteriormente dejará plasmados en sus libros de Urología.

Palabras clave: Historia de la Urología. Dr. Puigvert Gorro. Biografía Dr. Puigvert

Summary.- OBJECTIVES: For the celebration of the first Century of Dr. Antonio Puigvert Gorro's birth we want to know his contribution to and relationship with Archivos Españoles de Urología.

METHODS: We review all articles published by Dr. Puigvert, and additionally all articles with reference to his person and his work; we also review his written works and biography.

RESULTS: He was one of the founders of the journal and always maintained a great cooperation until the '70s; he published important works in it, which are analyzed. We also emphasize his contribution to Urology in addition to his dedication to the history of the speciality.

CONCLUSIONS: Dr. Puigvert is one of the most distinguished figures in the Spanish Urology, with an immense working capacity and written works. In his works published in Archivos he presents the results of his observations and his studies, as well as part of his innovations, ideas, and discoveries, that he later left in his urology books.

Keywords: History of urology. Dr. Puigvert Gorro. Dr. Puigvert biography. 


\section{INTRODUCCIÓN}

El 26 de abril de 2005 se cumplió el centenario del nacimiento del Dr. D. Antonio Puigvert Gorro (1905-1990), Figura 1, y como discípulo suyo, he repasado su vida, su obra y su contribución al auge de nuestra especialidad. Quiero destacar, también, su relación con Archivos Españoles de Urología y el importante papel que esta publicación desempeñó en el restablecimiento de la actividad científica española, desaparecida a causa de la Guerra Civil, ya que ocupó el vacío existente y ayudó a que los urólogos españoles contaran con un medio de expresión donde pudieran dar a conocer sus trabajos. Le corresponde al Dr. D. Enrique Pérez Castro (1908-1980) el mérito de ser el promotor y organizador de la revista, reunió a las principales personalidades urológicas del momento y les expuso su idea de editar una publicación de contenido netamente urológico; aceptaron sin la menor objeción los Drs. D. Luis Cifuentes Delatte (1907-2005) y D. Antonio Puigvert y, juntos, acordaron que llevase el nombre de Archivos Españoles de Urología. En el primer número, aparecido en julio de 1944, declaran sus intenciones en la presentación titulada «Primeras Palabras», cuyo sentido explicará el Dr. Cifuentes al celebrarse el $\mathbf{4 0}$ aniversario: significaba el primer paso de nuestra deseada realización.

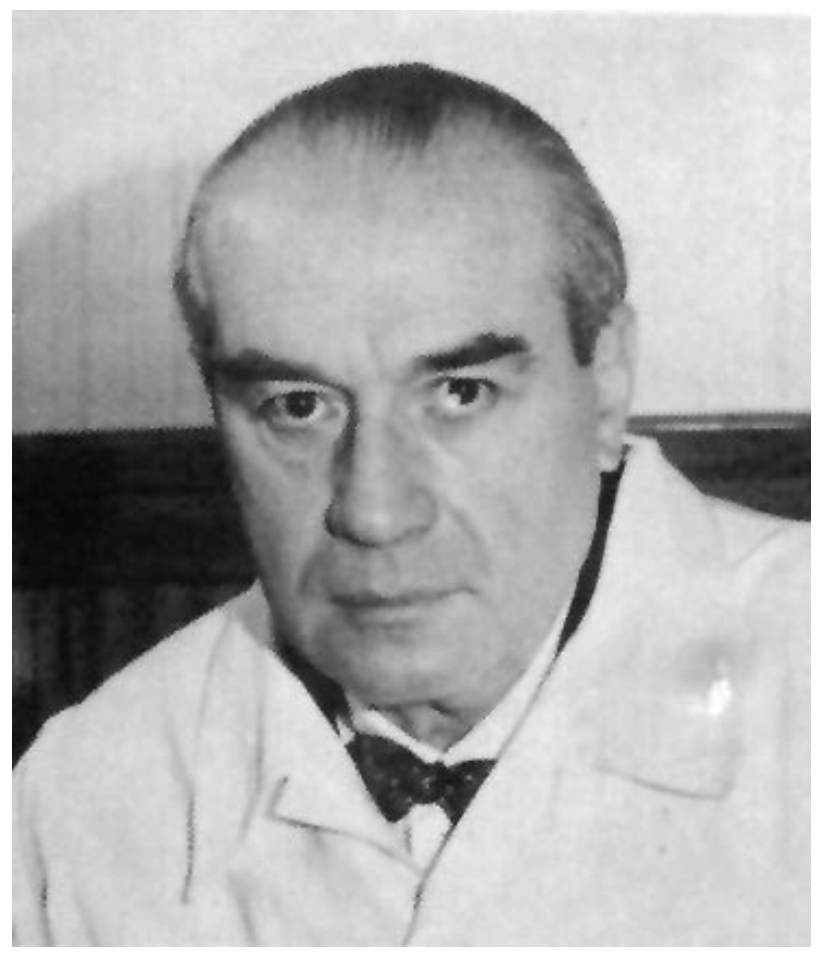

FIGURA 1. Dr. Puigvert.
Nuestros Archivos son una publicación totalmente nueva e independiente, nacida para servir a la Urología española... nuestros Archivos responderán fielmente a su título... quedarán archivados absolutamente todos los artículos de Urología que se publiquen en nuestra Patria...

Por espacio de cerca de treinta y tres años, hasta la aparición de Actas Urológicas Españolas en enero de 1977, fue la publicación urológica española de mayor relevancia y, fieles a su propósito, encontramos recogido entre sus páginas el trabajo de los compañeros que nos antecedieron gracias a lo cual nos muestra el camino recorrido y la evolución que ha llevado la Urología y, aún hoy día, tras de más de seis décadas, mantiene el espíritu que su fundadores le inculcaron y desempeña un trascendente papel en la bibliografía urológica hispana. En su apartado de «noticias» informa puntualmente de los acontecimientos más importantes de la Urología y de los que protagonizan los principales urólogos españoles, lo que la convierte en el único y fiel testimonio de esa época de nuestra historia, de la que el Dr. Puigvert es un activo protagonista.

Al repasar sus aportaciones en Archivos nos percatamos que su gran inteligencia, su agudo ingenio y su perspicacia le hicieron intuir la valía y la trascendencia que la publicación tenía y aun la que adquiriría con el paso de los años, especialmente el impacto que tendría fuera del país, sobre todo en Hispanoamérica, por lo que encontramos que sus artículos originales recogen, por lo general, el contenido de las conferencias dadas en sus periplos por dicho continente; además son constantes las referencias tanto de sus viajes, asistencia a Congresos, charlas y aportaciones urológicas, como del desarrollo de su Instituto de Urología y de los cursos monográficos de formación de postgrado en él impartidos así como de los llevados a cabo en el Sudamérica, todo ello nos ayuda a conocer un amplio retazo de la vida.

Dentro de las características de su personalidad destacan, ya desde su juventud, su profundo amor al estudio y su intensa dedicación a su preparación científica y quirúrgica lo que, tras su tras licenciatura en 1928, trasmite al conocimiento de la patología urológica; ya en sus primeros libros nos lo demuestra, en la redacción de su Atlas de Urografía de 1933, reseña 760 trabajos consultados y a su tratado de Endoscopia urinaria, de 1939, le acompaña un millar de citas bibliográficas, de tal modo que, cuando en 1954 traslada su Instituto de Urología al Hospital de la Santa Cruz y de San Pablo de Barcelona, se lleva, además de su extensa biblioteca, un completo fichero que incluye unas 70.000 referencias de artículos urológicos; otro aspecto de 
su genialidad lo constituye su claridad de ideas que le hizo conservar todas las historias clínicas de los pacientes asistidos con el contenido de las exploraciones analíticas, endoscópicas, radiográficas, etc. practicadas, y, para escribir su obra, La tuberculosis urinaria y genital masculina, en 1958, se apoya en su archivo personal de más de 25.000 enfermos.

Otra faceta suya es la de reunir en su entorno a una serie de colaboradores que le ayudan en sus fines, entre los que destacamos al dibujante $D$. Antonio Belenguer, quien recoge con fiabilidad apuntes que resaltan los detalles de sus intervenciones qui-
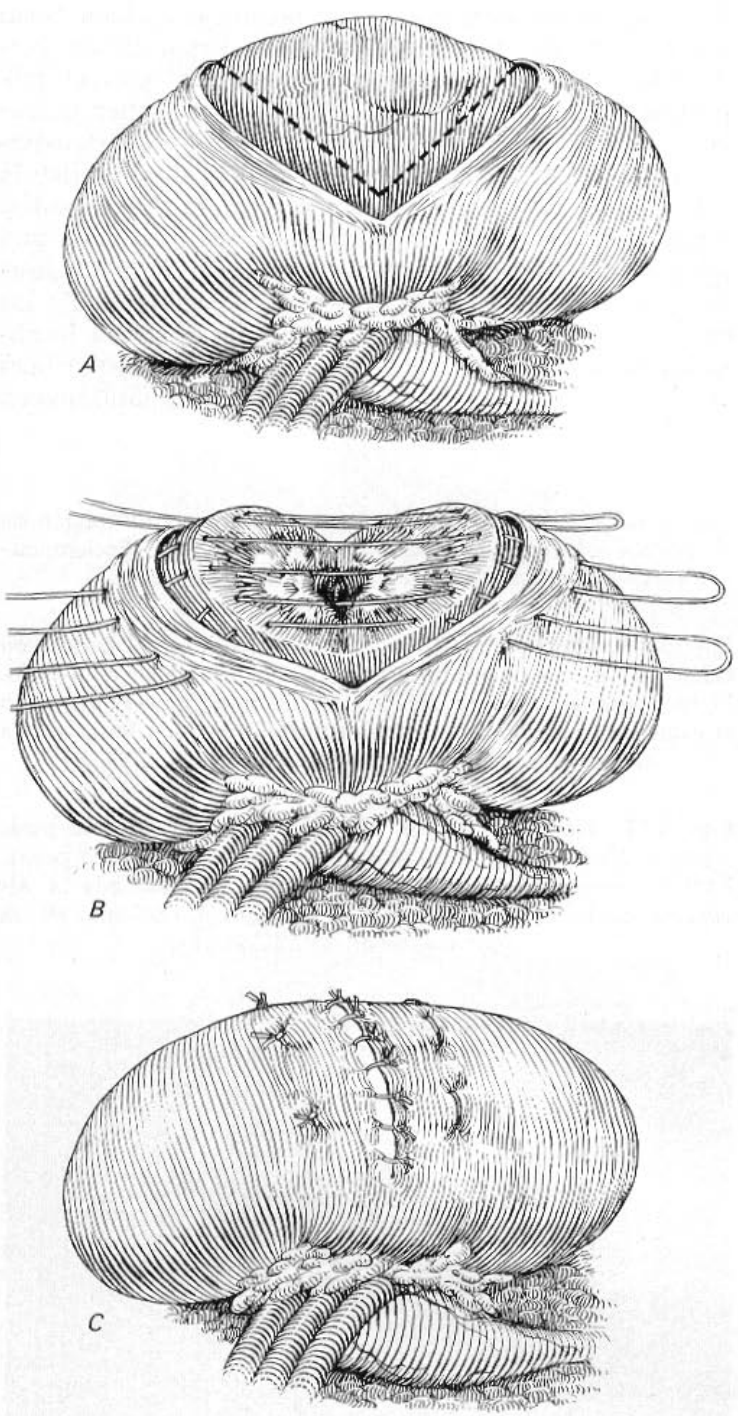

Fig. 8.18 Nefrectomía parcial del tercio medio.

FIGURA 2. Esquema de una nefrectomía parcial de tercio medio, dibujado por el Sr. Belenguer. rúrgicas, bocetos que alcanzaron la cifra de cinco mil y cuya selección podemos hallar plasmada en las magníficas láminas que acompañan a sus obras, Figura 2.

Desde que comenzó su andadura la revista hasta el año 1988 encontramos, en los cuarenta y un tomos editados en esos 44 años, 50 artículos originales firmados por el Dr. Puigvert, algunos con colaboradores, en los primeros años su participación es abundante en los últimos algo esporádicas, pero su apoyo a la publicación se mantendrá hasta el final de sus días. Analizadas sus aportaciones en su conjunto, permiten establecer su sistema de trabajo y que, a su vez, nos sirven de lección y ejemplo de cómo se deben redactar. Principia con un extenso y tan bien documentado análisis histórico de la patología objeto de estudio que sorprende por su amplia y completa revisión bibliográfica y que enriquece de modo notable su aportación; prosigue su exposición escrita con una detenida explicación etiopatogénica y con la descripción del o de los casos clínicos, efectúa unas consideraciones justificativas de los medios de exploración precisos y de los utilizados para llegar al diagnóstico, continúa con el proceder quirúrgico, que describe con minuciosidad, $y$, por lo general, acompaña de magníficos dibujos realizados por sus colaboradores, después enfoca el estudio anatomopatológico junto a la fotografía de la pieza extirpada y de las preparaciones microscópicas en
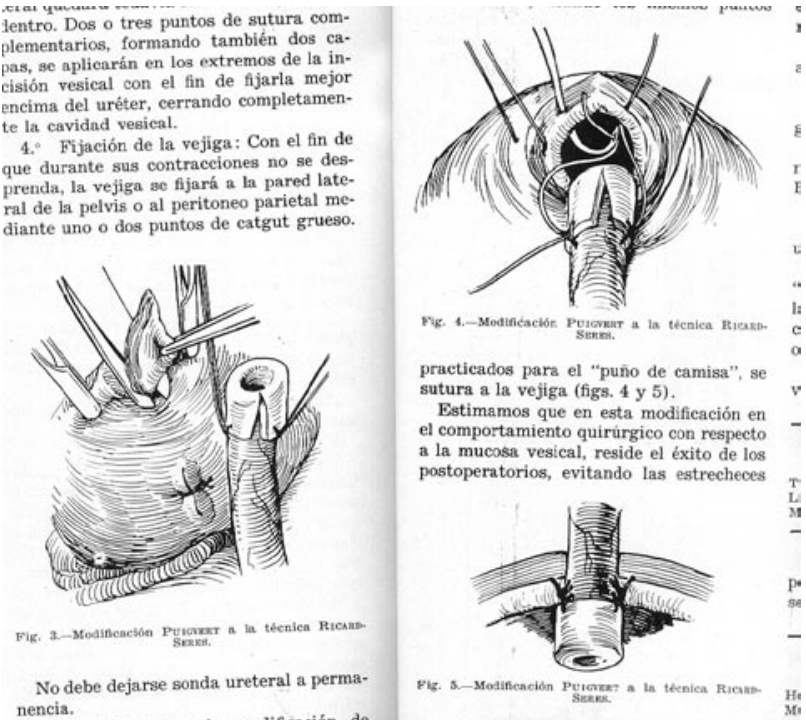

FIGURA 3. Reimplante ureterovesical, técnica de Puigvert. 
las que razona los hallazgos encontrados que justifican el diagnóstico y que, en ocasiones, recoge en diagramas para su mejor comprensión; finaliza, tras apuntar la evolución y las posibles complicaciones, con unas reflexiones generales o conclusiones en las que resaltan su saber y su buen juicio clínico.

De este modo destaca, en los trabajos editados en Archivos, su contribución al estudio de la nefrectomía parcial, (Figura 4), que, ya en 1929, en sus inicios como cirujano, practica y que la considera indicada en casi todos los casos de afecciones renales cuya lesión se encuentra circunscrita a una zona del órgano, con ello se significa como uno de los primeros en abordar esta intervención en casos de tuberculosis y en la exéresis del foco litógeno en la litiasis renal, además de efectuarla en los tumores renales a partir de 1954 y sobre la que señala la importancia de conocer la vascularización renal, previa a la cirugía, por medio de una aortografía, según la técnica descrita por el portugués Dr. Reynaldo Dos Santos; en su publicación, de 1960, presenta su casuística de 198 enfermos intervenidos de nefrectomía parcial y en la de 1988, comenta 33 casos intervenidos por tumor con diez años de supervivencia. La patología urológica de la tuberculosis renal y su tratamiento quirúrgico centran principalmente sus estudios, con 24 artículos en los que describe las estenosis yuxtavesicales y en los que hace hincapié en la cirugía temprana como prevención del deterioro de la función renal a consecuencia de la dilatación ureteral; señala cinco diferentes métodos de reimplante ureterovesical entre ellos el extravesical en «puño de camisa», según la técnica de Ricard-Serés modificada por él, (Figura 3); en las esclerosis piélicas de etiología fímica la opción que propone, en 1965, como solución en lugar de la nefrectomía, es la uretero-cálico-anastomosis, otra de sus innovaciones quirúrgicas. En todo ello sobresale su criterio conservador renal en contra de la extirpación precoz imperante y su decidida actuación sobre la estenosis ureteral tuberculosa no considerada como alternativa por la mayoría de autores en aquellos tiempos.

Su aportación a la cirugía de las hidronefrosis congénitas con la práctica de una Ureteropie-

\begin{abstract}
ARCHIVOS ESPAÑOLES DE UROLOGIA TOMO XIV Deposito legal M. 9.003-1063 NÜM. 2 PUBLICADO POR E. PEREZ CASTRO, A. PUIGVERT gORRO Y L. CIFUENTES delatie

Instituto de Urologia. Hospital de ln Santa Cruz y San Pablo. Barcelon. Director: Dr. A. PUIGverT.

LA NEFRECTOMIA PARCIAL (")

A. PUigvert.

Han transcurrido más de catorce años desde aquella mañana, de gratísimo recuerdo para mi, en que en esta misma Cátedra de Urología, entonces regentada por mi dilecto amigo el profesor E. CAsTaÑo, desarrollé la primera conferencia en tierra argentina acerca de la Nefrectomía Parcial. Fundamentó la disertación el acoplo de datos bibliográficos, enseñanzas ajenas y la corta casuistica de 14 casos. Por el día de hoy aquel tema, presentando mi actual experiencia, de mayor importancia aquel tema, presentando mi actual experiencia, de mayor importancia numérica, y las deducciones, en esta ocasión personales, que en estos años he alcanzado. En realidad, este tema constituye la justificación y comprobación a las tesis sustentadas en aquella ocasión y que de nuevo presento en esta misma Cátedra, en la actualidad regentada por mi buen presonto en la presencia señera del doctor Castaño.

En los últimos años la práctica de la N. P. se ha extendido con sin-

En los últimos años la práctica de la N. P. se ha extendido con sin-
alar atención y naturales aportaciones en su mejoramiento técnico, en todos los centros de Urología, siendo además tema de estudio y discugión en algunos de los últimos Congresos de Urología. Con ello ha cusion en algo conduaumentado la casuistica y surge más importante ha cido a la mejora de los resultados; pero el avance más importante ha sido la ampliación de sus indicaciones, como puede comprobarse en el cuadro adjunto, que resume la actual casuística de $198 \mathrm{~N}$. P. realizadas

(*) Conferencia en la Catedra de Urologia de la Universidad de Buenos Aires, septiembre de 1958.
\end{abstract}

FIGURA 4. Conferencia sobre nefrectomía parcial, impartida en Buenos Aires en 1958.

\section{ARCHIVOS ESPAÑOLES DE UROLOGIA \\ TOMO XVII Deposito legal M. 2.003-1958 NÚM. publicado por e. perez Castro, a. puigvert gorro y L. Cifuentes delatite}

Instituto de Urologia, Hospital de la Santa Cruz y San Pablo. Barcelona Director: Dr. A. PUIGVERT.

HIDRONEFROSIS E HIDROCALICOSIS (")

A. Puigvert.

La hidronefrosis, a excepción de aquellas de origen congénito, "no es una enfermedad", es un signo morfológico común a muchas enfermedades, resultante de la obstrucción de las vías excretoras del riñón. La obstrucción puede hallarse a partir del ostium ureteral hasta la uretra, y sea donde fuese, su acción puede alcanzar al rińón.

Toda dificultad que se oponga al libre tránsito de la orine por las vias urinarias, invirtiendo el coeficiente de evacuación, con relación al de secreción renal, dará lugar al remanso constante y progresivo de orina en las vias urinarias, que obliga a la dilatación de la pelvis, eálices o de todas las vías urinarias, según y donde se encuentre el obstáculo.

Cuando el obstáculo está en el ostium ureteral, da lugar a la dilatación pielocalicilar, conocida con el nombre de hidronefrosis, con que RAYER en 1841 bautizó esta afección en su libro "Enfermedades del riñón". Cuando la obstrucción es en el meato ureteral provoca la uréterohidronefrosis; cuando el obstáculo está en el cuello calicilar produce hidrocáliz.

En realidad, la primera observación anatomoclínica de dilatación pielocalicilar, se debe al médico holandés Nrcor ís TuLPIUS, en un paciente que aquejaba crisis dolorosas abdominales, en el cual la necropsia descubrió la pelvis renal enormemente dilatada y rellena de orina, sia descubrió la pelvis renal enormemente dilatada y rellena de orina, a semejanza de la vejiga urinaria. TuLPIUs relacionó el hallazgo anatoficado de "ischuria renal lunática".

(*) Conferencia en la Universidad de Salamanea. I Curso Báslco de Urologia Salamanca, 9 de febrero de 1963.

FIGURA 5. Hidronefrosis e hidrocáliz, conferencia dada en Salamanca en 1963. 
loplastia la presenta en sendos artículos en 1956 y en 1978, en ellos cuestiona algunas de las teorías etiopatogénicas y apunta la propia de que se origina debido a factores isquémicos ocurridos en el desarrollo; señala también la conveniencia de reducir la resección piélica y de centrarse en la extirpación amplia de la zona estenótica ureteral. Desde 1963 estudia la abundante iconografía que acumula el Instituto de Urología con 180.000 placas radiográficas de distintas técnicas y de su análisis descubre una nueva patología, la «Megacaliosis», con la que define la dilatación congénita pielocalicilar no obstructiva como consecuencia de un desarrollo incompleto de los tubos colectores de Bellini, en Archivos presenta su primera comunicación sobre ella en 1964, Hidronefrosis e hidrocalicosis, Figura 5, a la que siguen otras tres y sobre la que escribe dos libros, Malformaciones congénitas de la pirámide renal de Malpighi, en 1965, y Semiología medular del riñón. Estudio clínico y radiográfico, en 1981.

La mayoría de los trabajos que encontramos en Archivos, y por los que muestra gran predilección,

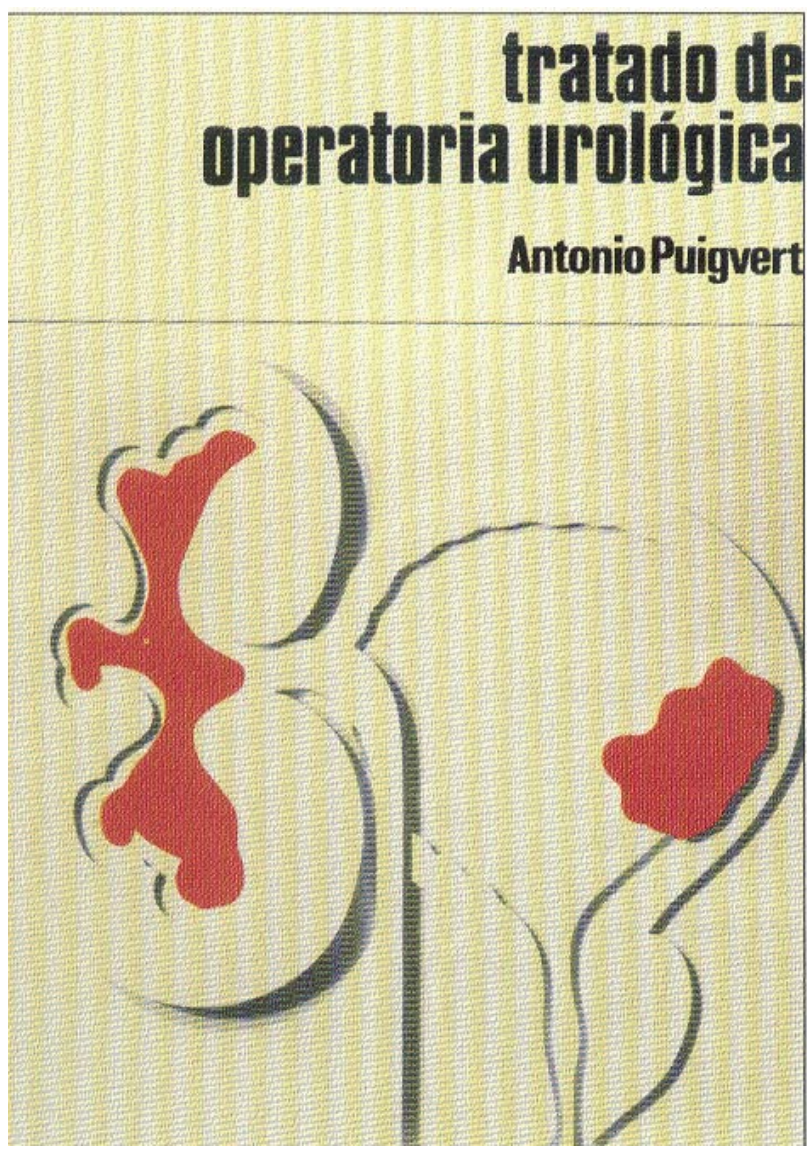

FIGURA 6. Tratado de Operatoria Urológica. son en los que expone las técnicas quirúrgicas, de un modo ordenado, paso a paso, acompañadas de sus diagramas y dibujos para su mejor comprensión, así, además de los referidos anteriormente, describe sus aportaciones a la adenomectomía por vía perineal, a la operación de Boari-Casatti, a la de iliocistoplastia, a una variante personal de la técnica de Delinotte para el tratamiento de la incontinencia urinaria femenina y a la orquidofuniculolisis principalmente. En 1971 vio la luz su Tratado de Operatoria Urológica, Figura 6, en el que concentra todo el material que, con su gran experiencia y práctica, había acumulado a lo largo de los años, con sus métodos y su modo de hacer cirugía, intervenciones nuevas o enriquecidas con modificaciones personales, junto a su espléndida iconografía, ante la solicitud de sus discípulos, amigos y colaboradores que le pedían que las reuniese en un libro.

Los casos clínicos que nos presenta corresponden a patologías muy poco frecuentes, raras y con escasa casuística bibliográfica en el momento, como el Diagnóstico clínico de un aneurisma de arteria renal o Herida en cava o su contribución al estudio de la Ureteritis quística, entidad pobremente descrita hasta ese momento. En el resto de trabajos siempre encontramos innovaciones personales, como la exploración intradiverticular endoscópica en los divertículos vesicales o las aplicaciones de la cistovesiculografía - la uretrografía, en los que apunta sus indicaciones precisas al estilo de sus tratados de Endoscopia urinaria y de Urografía clínica.

Una muestra de su sorprendente personalidad la tenemos en su afición y su dedicación a la historia de la Medicina, sobre la que, a lo largo de su carrera, redactará numerosos artículos entre los que destacamos, en primer lugar, los que escribió sobre Francisco Díaz, el insigne urólogo del siglo $\mathrm{XVI}$ y su obra, Enfermedades de los riñones, vexiga

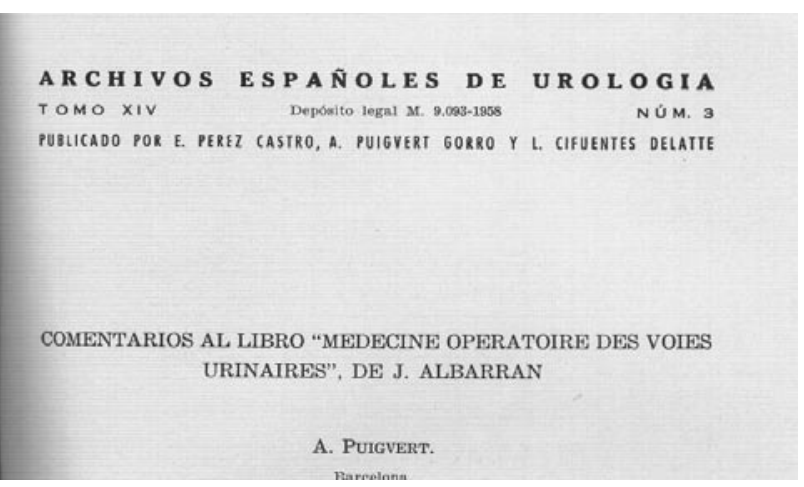

FIGURA 7. Comentarios al libro de Albarrán. 
y carnosidades de la verga y urina by Francisco Díaz. Coment on an old book, en 1978, que hizo que se lo reconociera internacionalmente como «Padre de la Urología», y sobre su Tratado, el primero en el mundo escrito exclusivamente sobre la especialidad, del que patrocinó una edición facsímil en 1968; en la memoria del autor creó, en 1972, una medalla con su nombre, que se otorga anualmente a la persona que más haya destacado por su contribución y dedicación a la Urología; su faceta de biógrafo aflora en sus análisis de la obra y de la vida de Joaquín Albarrán (1860-1912), por quien sentía una gran admiración y respeto, reflejados en cuatro diferentes trabajos, Comentarios a un libro de Urología de 1909, en 1959, Comentario al libro Medicine Operatoire des voies urinaires, de Joaquín Albarrán, Figura 7, en 1960, Un médico hispano cubano en "la Sorbona", Dr. J. Albarrán y La obra científica de J. Albarrán (1885 a 1912) ambas en 1961; efectuó también semblanzas de urólogos tanto clásicos como de prestigio internacional, o la de los amigos y compañeros recién fallecidos, de los que, en la revista, encontramos varios ejemplos; además de, como ya hemos indicado realizar una amplia revisión histórica de las patologías por él estudiadas como ejemplos su Visión histórica de la tuberculosis uro-genital, de 1957, su excelente estudio sobre los denominados Fungus vesicales, en 1965, y, por último, su libro El mal de piedra del riñón en 1987, como los más señalados.

Su colaboración en Archivos representa una pequeña muestra de su inmensa capacidad de trabajo, cuyo exponente más fiel lo hallamos en las Series del Instituto de Urología, cuadernillo editado en este centro, tras el traslado al Hospital de la Santa Cruz

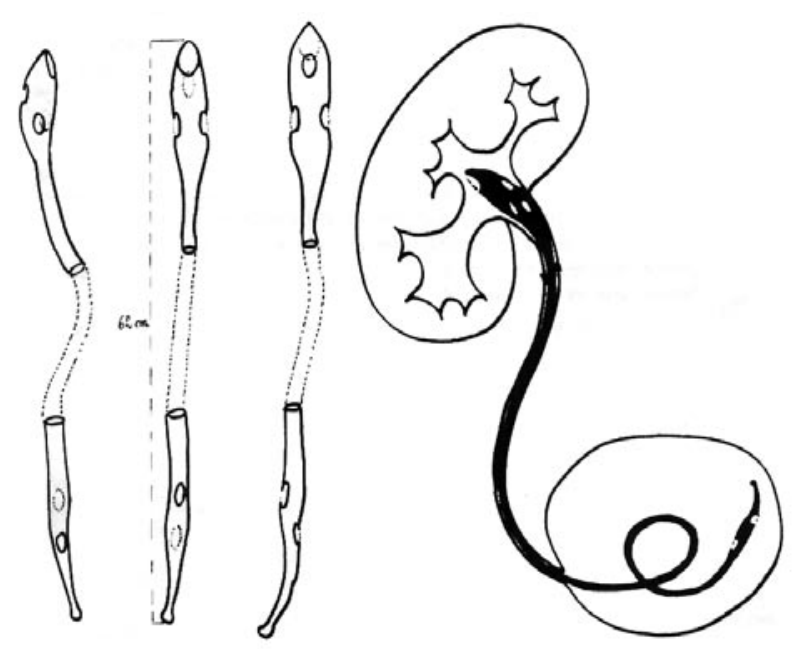

FIGURA 8. Sonda tutor ureteral en cabeza de cobra de Puigvert. y San Pablo de Barcelona de su clínica en 1954, en el que anualmente se recogen grapadas separatas de todos los artículos urológicos publicados por los miembros de la institución dirigida por él y donde podemos valorar su labor ya que, en las 23 series que completan la edición hasta 1970, recoge 146 trabajos con su firma, todos ellos originales, un promedio anual de cerca de nueve artículos, de ellos 23 en revistas internacionales, principalmente en lengua francesa, lo que nos da una idea de su extensa labor publicista.

No puedo por último olvidar las referencias que sobre su persona y obra he localizado en la publicación, todas ellas en 1991, en un número conmemorativo con ocasión de su fallecimiento, una Carta del editor, del Dr. Pérez-Castro Ellendt, dedicado a su recuerdo, el artículo del Dr. Solé Balcells, Antonio Puigvert. El choque de "pareceres»: un avance en el reconocimiento, en relación con sus enseñanzas a las nuevas generaciones de urólogos, del Dr. Villavicencio, La estela de la Urología de Antonio Puigvert, en la que expresa su admiración por su persona y por su obra, del Dr. Rioja Sanz, Recuerdo. Antonio Puigvert, narración de su primer encuentro y de la impresión que le produjo, del Dr. Vela Navarrete, Cirugía reconstructivas pieloureteral en la obra de Antonio Puigvert, en la que, junto a Drenajes, nefrostomías y tutores en la cirugía reconstructiva del tracto urinario superior, de 1982, recoge su contribución quirúrgica en la cirugía renal especialmente tuberculosa y su sonda tutor ureteral en cabeza de cobra autorretenible, Figura 8, para el cateterismo interno prolongado de la vía excretora, precursora de los actuales catéteres doble J.

Mucho más podría hablar de sus relaciones y de su trato personal, de su sensibilidad o de sus, a veces, geniales aportaciones, pero he preferido centrarme en el papel que representó su colaboración en la Archivos Españoles como un homenaje a su contribución al mayor esplendor de la publicación de este catalán de pensamiento y sentimiento, gran figura de la Urología española.

\section{Relación de los trabajos del Dr. Puigvert publicados en Archivos Españoles de Urología:}

«Nuevas aportaciones a la adenomectomía por vía perineal», I (1): 22-36, 1944.

«Nefrectomía parcial», II (1): 29-49, 1945.

«Divertículos vesicales. Aportación a su diagnóstico y tratamiento», II (3): 263-285, 1945.

«Tuberculosa pararrenal y tumor de pelvis renal», III (1): 14-29, 1946.

«Aneurisma de la arteria renal», III (4): 203-211, 1946. 
«Cistovesiculografía simultánea», IV (2): 135-142, 1947.

«Cáncer y tuberculosis de riñón», IV (3): 200-219, 1947.

«Herida de la vena cava durante la nefrectomía», V (1): 66-76, 1948.

«Uretrografía en los operados de adenomectomía perineal», V (2): 104-110, 1948.

«Ureterosigmoidostomía extraperitoneal», VI (2): $111-127,1949$.

«La ureteritis tuberculosa estenosante terminal», VII (3): 183-203, 1951.

«Mi experiencia en nefrectomía parcial por tuberculosis», IX (2): 101-115, 1953.

«Cuerpo extraño vesical», IX (4): 297-301, 1953.

«La operación de Boari-Casati», X (2): 101-115, 1954.

«La ileocistoplastia», XI (2): 89-102, 1955.

«Síntomas urológicos de los aneurismas de la aorta abdominal», XI (2): 136-148, 1955.

«Tumor sigmoideo vesical», XI (4): 245-250, 1955.

«La ureteropieloplastia», XII (3): 135-158, 1956.

«Tratamiento de la incontinencia urinaria femenina», XIII (1): 1-10, 1957.

«Hernia vesical inguinal», XIII (2): 85-90, 1957.

«Divertículo de la uretra femenina», XIII (3): 173-190,

1957.

«La nefrectomía parcial», XIV (2): 79-90, 1958.

«Tuberculosis preclínica y secundarismos», XIV (2): 144-152, 1958.

"Comentarios al libro "Medicine opératoire des voies urinaires" de J. Albarrán», XIV (3): 159-186, 1958.

«Derivación prevesical de la orina», XV (4): 314 339, 1962.

"La orquidofuniculosis en el tratamiento de la criptorquidia», XVI (3): 197-211, 1963.

«Recuperación renal después de obstrucción ureteral», XVI (4): 275-313, 1963.

«Hidronefrosis e hidrocáliz», XVII (1): 1-17, 1964. «Prostatectomía transvesical hemostática», XVII (1): 185-201, 1964.

«Fundamentos arteriográficos en el tratamiento de la tuberculosis renal», XVII (1): 291-280, 1964.

«Ureterocaliostomía en el tratamiento de la tuberculosis renal la tuberculosis renal», XVIII (1): 1-8, 1965.

«La cirugía de sustitución en la tuberculosis urinaria. Resultados lejanos», XVIII (2): 92-106, 1965.

«Ureterocistorrafias. Experiencia de diez años», XIX (1): 1-14, 1966.

«Malformaciones congénitas de la pirámide renal de Malpighi», XIX (2): 101-120, 1966.

«Disembrioplasia médulo-tubular. Tuberculosis y litiasis», XIX (4): 339-348, 1966.

«Cirugía conservadora en los tumores de la pelvis renal», XX (3): 280-303, 1967.

«Diagnóstico radiológico diferencial entre hidronefrosis, displasias pielocalicilares y tumor del seno re- nal», XXI (3): 283-299, 1968.

«La nefrectomía parcial por tumor», XXII (1): 1-35, 1969.

«La ureteritis quística», XXIV (1): 1-35, 1973.

«Algo acerca de la ureteritis quística», XXVII (6): 639656, 1974.

«Problemática quirúrgica en la calculosis bilateral», XXX (1): 1-10, 1977.

«Metástasis en epidídimo de carcinoma de próstata», XXXI (1): 1-10, 1978.

«Comentarios a los tratamientos quirúrgicos de la hidronefrosis», XXXI (4): 313-337, 1978.

"40 aniversario de Archivos Españoles de Urología», 37 (1): 2, 1984.

«Infiltración intersticial en el riñón», XXXVI (2): 67-76, 1983.

«Endoscopia en general y urinaria», 37 (2): 651 659, 1984.

"Comentario clínico práctico», 38 (1): 79-81, 1985. «Poliquistosis medular del riñón», 39 (2): 92-100, 1986

«La historia clínica», 39 (8): 539-543, 1986.

«La nefrectomías parcial por tumor»,41 (7): 511 $515,1988$.

\section{BIBLIOGRAFÍA Y LECTURAS \\ RECOMENDADAS ( ${ }^{*}$ lectura de interés $y$ ** lectura fundamental)}

1. DÍAZ RUBIO, M.: "Antonio Puigvert i Gorro (1905-1990)". 100 médicos españoles del siglo XX, pág. 156, edit. You \& Us, Madrid, 2000.

*2. PÉREZ ALBACETE, M.: "Centenario del nacimiento del Dr. D. Antonio Puigvert Gorro". Actas Urol. Esp., 29: 807, 2005.

3. PÉREZ ALBACETE, M.: "100 Figuras de la Urología Española”. pág. 180, tip. San Francisco, Murcia, 2005.

*4. PUIGVERT GORRO, A.: "Atlas de Urografía". 215 págs., Salvat edit. Barcelona, 1933.

**5. PUIGVERT GORRO, A.: "Endoscopia urinaria". 228 págs., $1^{\text {a }}$ edic. Salvat edit. Barcelona-Buenos Aires, 1939.

6. PUIGVERT GORRO, A.: "Exploración radiográfica de los órganos urinarios durante el acto quirúrgico". Tesis doctoral, Madrid, 1941.

7. PUIGVERT GORRO, A.: "Tuberculosis génitourinaria”. 160 págs., Salvat edit. BarcelonaBuenos Aires, 1941.

*8. PUIGVERT GORRO, A.: "Tratado de urografía clínica". Salvat edit. Barcelona-Buenos Aires, 1944. 
*9. PUIGVERT GORRO, A.: "Tumores de la vejiga. Evolución y fundamentos de clasificación anatomo clínica". 60 págs., discurso de ingreso en la Real Academia de Medicina de Barcelona, Barcelona, 1952.

*10. PUIGVERT GORRO, A.: "Tuberculosis urinaria y genital masculina". 375 págs., Salvat edit. Barcelona-Madrid-Buenos Aires, 1958.

**11. PUIGVERT GORRO, A.: "Malformaciones de la pirámide renal de Malpighi”. 202 págs., ed. bilingüe español francés, edit. ECO S A., Barcelona, 1965.

**12. PUIGVERT GORRO, A.: "Endoscopia urinaria". $2^{\mathrm{a}}$ ed. 146 págs., edit. ECO S A, Barcelona, 1969. Reediciones edit. JIMS, SA., Barcelona, 1975 y 1983.

**13. PUIGVERT GORRO, A.: "Tratado de operatoria urológica”. 525 págs., edit. Labor S A, Barcelona, 1971.

14. PUIGVERT GORRO, A.: "Del saber y del hacer urológico". Conferencia de ingreso en la sociedad de médicos escritores, edit. ECO, SA, Madrid, 1976.

*15. PUIGVERT GORRO, A.: "Semiología medular del riñón. Estudio clínico y radiográfico". 185 págs., edit. Jims, Barcelona, 1981.

**16. PUIGVERT GORRO, A.: Atlas de Urografía." 1986, 566 págs., $2^{a}$ edición, edit. Salvat SA, Barcelona, 1986. Reedición 1989.

*17. PUIGVERT GORRO, A.: "Mal de piedra del riñón”. Salvat SA, edit., Barcelona, 1987.

18. RIOJA SANZ, L.Á.: "Recuerdo. Antonio Puigvert”. Arch. Esp. Urol., 44: 339, 1991.

*19. ROUSAUD BARÓN, A.; PONCE DE LEÓN ROCA, J.; GAUSA GASCÓN, L. y cols.: "El Dr. Antonio Puigvert y su obra". Historia Biográfica y Bibliográfica de la Urología Española en el siglo XX, págs. 185-194, Edicomplet, Madrid, 2002.

20. SOLÉ BALCELLS, F.J.: "Antonio Puigvert. El choque de pareceres". Arch. Esp. Urol., 44: 333, 1991.

21. VELA NAVARRETE, R.: "Drenajes, nefrostomías y tutores en la cirugía reconstructiva del tracto urinario superior". Arch. Esp. Urol., 35: 263, 1982.

*22. VELA NAVARRETE, R.: "Cirugía reconstructivas pieloureteral en la obra de Antonio Puigvert". Arch. Esp. Urol., 44: 343, 1991.

*23. VILLAVICENCIO MAVRICH, H.: "La estela de la Urología de Antonio Puigvert". Arch. Esp. Urol., 44: 337, 1991. 